\title{
A Terahertz Blackbody Radiation Standard Based on Emissivity Measurements and a Monte-Carlo Simulation
}

\author{
C. Monte • B. Gutschwager • A. Adibekyan • J. Hollandt
}

Received: 10 October 2013 / Accepted: 31 January 2014/

Published online: 19 February 2014

(C) The Author(s) 2014. This article is published with open access at Springerlink.com

\begin{abstract}
Blackbody radiators are commonly used metrological standards of spectral radiance and radiation temperature according to Planck's law of thermal radiation. In a well defined geometry of observation they also provide calculable irradiance for the calibration of radiation detectors. Here we describe the metrological characterization of a vacuum variable-temperature blackbody to serve as a source standard for FIR- and $\mathrm{THz}$ radiation from $5 \mu \mathrm{m}$ to $200 \mu \mathrm{m}(60 \mathrm{THz}$ to $1.5 \mathrm{THz})$. The key quantity of the characterization is the effective spectral emissivity of its cavity. This is determined by angular resolved directional spectral emissivity and directional spectral reflectivity measurements of its wall coating, Aeroglaze Z306. From the reflectivity measurements the diffusity is determined. Spectral emissivity and diffusity in combination with the well known cavity geometry allow the determination of the effective spectral cavity emissivity via a Monte-Carlo ray tracing simulation.
\end{abstract}

Key words $\mathrm{THz} \cdot$ Blackbody $\cdot$ Emissivity $\cdot$ Effective emissivity $\cdot$ Aeroglaze Z306

\section{Introduction}

The knowledge of the absolute irradiance responsivity of detectors is crucial for the design and evaluation of quantitative optical experiments and applications in all spectral ranges. In the FIR- and THz range this information is often lacking. A direct approach to determine the irradiance responsivity of a FIR- and THz detector system is by applying calculable blackbody radiation provided by a dedicated FIR- and THz blackbody [1-3]. As the blackbody emits a continuous spectrum from the UV- to the $\mathrm{THz}$ spectral range it has to be operated with different sets of well characterized FIR- and THz band- and longpass spectral filters in order to obtain spectrally resolved information in the relevant spectral range [3]. With these filters and a well defined geometry of observation the blackbody is operated as a calculable source of spectral irradiance for the direct calibration of detectors without an imaging system. Another field of application of FIR- and

C. Monte $(\bowtie) \cdot$ B. Gutschwager $\cdot$ A. Adibekyan $\cdot$ J. Hollandt

Physikalisch-Technische Bundesanstalt, Abbestraße 2-12, 10587 Berlin, Germany

e-mail: Christian.Monte@ptb.de 
$\mathrm{THz}$ blackbodies is as source standards of spectral radiance, to provide calibrations for imaging and spectrally resolving detection systems, for example in remote sensing applications.

Mandatory for the application of a blackbody as a radiometric standard is its precise metrological characterization regarding the absolute temperature, temperature homogeneity and effective spectral emissivity of its cavity. Due to the lack of well characterized and sufficiently black coatings in the FIR- and $\mathrm{THz}$ spectral range the knowledge of the effective cavity emissivity is the crucial point of an FIR- and THz source standard. In this paper we deal with this problem by determining the directional spectral emissivity and reflectivity of the cavity coating in a first step. The geometry of the applied blackbody is appropriate for long wavelength applications and its dimensions are well known. On the background of this sound data the effective spectral cavity emissivity is determined by a Monte-Carlo simulation [4]. Knowing the cavity's effective emissivity and, additionally, its temperature and possibly temperature inhomogeneity traceable to the International Temperature Scale (ITS-90) with the appropriate uncertainties its radiance can be reliably calculated according to Planck's law of thermal radiation.

In detail we outline this procedure for a dedicated vacuum variable-low-temperature blackbody (VLTBB) [5] applied from $-170{ }^{\circ} \mathrm{C}$ to $170{ }^{\circ} \mathrm{C}$ for remote sensing calibrations as part of the Reduced Background Calibration Facility (RBCF) of PTB [6]. The blackbody is coated with Aeroglaze Z306. A space-qualified coating which is often used as a high emissivity paint applicable under vacuum and in the low-temperature regime for blackbodies in the NIR- and MIR spectral range. The directional spectral emissivity of the wall coating was determined in a wavelength range from $5 \mu \mathrm{m}$ to $200 \mu \mathrm{m}$ corresponding to a frequency range from $60 \mathrm{THz}$ to $1.5 \mathrm{THz}$. Furthermore, the spectral reflectance was determined in this range which allows the calculation of the spectral diffusity. Finally, wall emissivity and diffusity were used in a Monte-Carlo simulation to calculate the effective emissivity of the blackbody.

\section{Experimental}

\subsection{Setup for Directional Emissivity Measurement}

PTB operates two instrumentations for directional spectral emissivity measurements in air [7] and under vacuum [8]. Both instrumentations work with the same measurement principle. For the characterization of the coating Aeroglaze Z306 the instrumentation under vacuum was applied, as it allows emissivity measurements in a wavelength range from $4 \mu \mathrm{m}$ to $200 \mu \mathrm{m}$ without atmospheric absorptions. Following the definition of the spectral emissivity the measurement is performed by measuring the spectral emission of the sample under investigation in direct comparison to the spectral emission of a reference blackbody radiator. Both measurements are performed sequentially by a Vacuum-Fourier-Spectrometer of type Bruker Vertex $80 \mathrm{~V}$ with identical equipment and under the same geometry of detection for both sources. By knowing the temperatures of the blackbody, the sample surface, the surrounding of the sample and of the detector traceable to the ITS-90 the emissivity of the sample can be calculated from the complete radiation budget. Furthermore, a second reference blackbody of significantly different temperature from the first reference blackbody is generally applied for the correction of the background signal. The instrumentation and the associated uncertainties of this measurement principle are published in detail in [7-9], respectively. To determine the 
angular dependency of the directional spectral emissivity from a series of the above described ratio measurements the sample can be tilted in a range from $5^{\circ}$ to $70^{\circ}$ between the sample surface normal and the direction of observation.

\subsection{Setup for Directional Reflectivity Measurement}

The directional spectral reflectance of the Aeroglaze samples was determined in a $12^{\circ} / 12^{\circ}$ geometry by using a commercial accessory for a Vacuum-Fourier-Spectrometer of type Bruker Vertex $80 \mathrm{~V}$. A sketch of the measurement instrumentation is shown in Fig 1. A gold mirror served as the reference sample. Depending on the investigated spectral range two sets of equipment were used: a pyroelectric detector of type DLaTGS with a potassium bromide window and a GLOBAR source in combination with a $\mathrm{KBr}$ beam splitter were used from $5 \mu \mathrm{m}$ to $16.7 \mu \mathrm{m}$ and a pyroelectric detector of type FDTGS with a polyethylene window and a GLOBAR source in combination with a $6 \mu \mathrm{m}$ Multilayer Mylar beam splitter were used from $16.7 \mu \mathrm{m}$ to $200 \mu \mathrm{m}$. Samples with a medium or high reflectivity are measured in a scheme where possible effects due to background radiation and sample emission are reduced. A more thorough description of the measurement scheme is given in [10].

\section{Results for Aeroglaze Z306}

\subsection{Directional Spectral Emissivity}

Samples with different coating thicknesses were investigated with the instrumentation described in section 2.1. The coating Aeroglaze Z306 was sprayed on a set of three copper substrates according to the instructions given in the European Cooperation for Space Standardization document ECSS-Q-70-25A [11] but without applying the primer. The number of cross layers sprayed was increased according to the document to obtain coatings with larger thickness. Finally, samples with coating thicknesses of $44 \mu \mathrm{m}, 99 \mu \mathrm{m}$ and $236 \mu \mathrm{m}$ were obtained by this. The measured directional spectral emittance of the

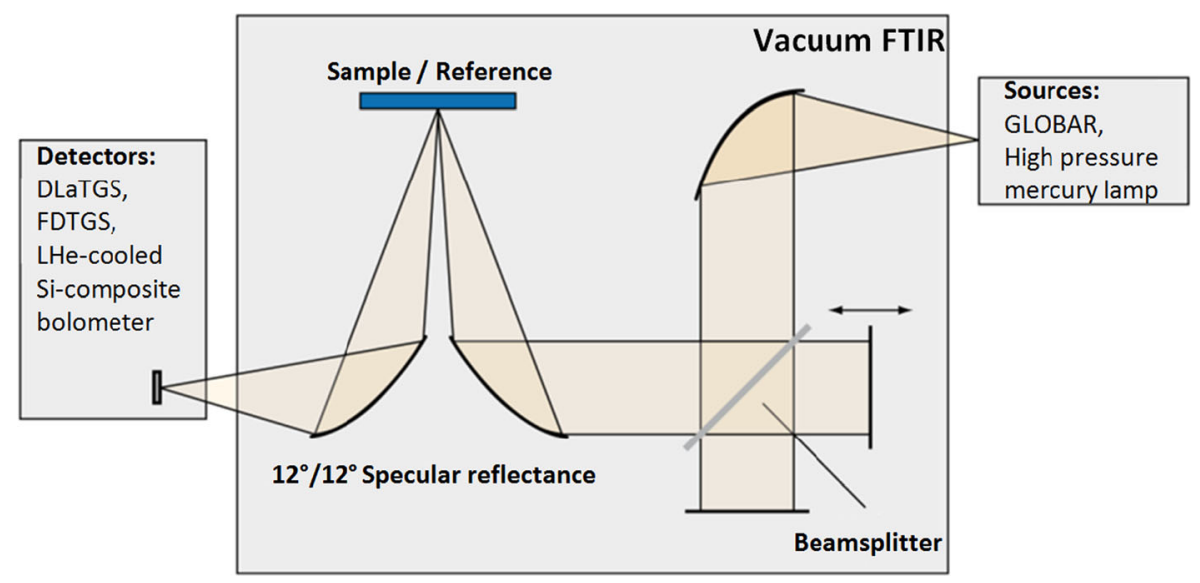

Fig. 1 Schematic of the setup for the determination of the directional spectral reflectivity of samples with a Vacuum-Fourier-Spectrometer 


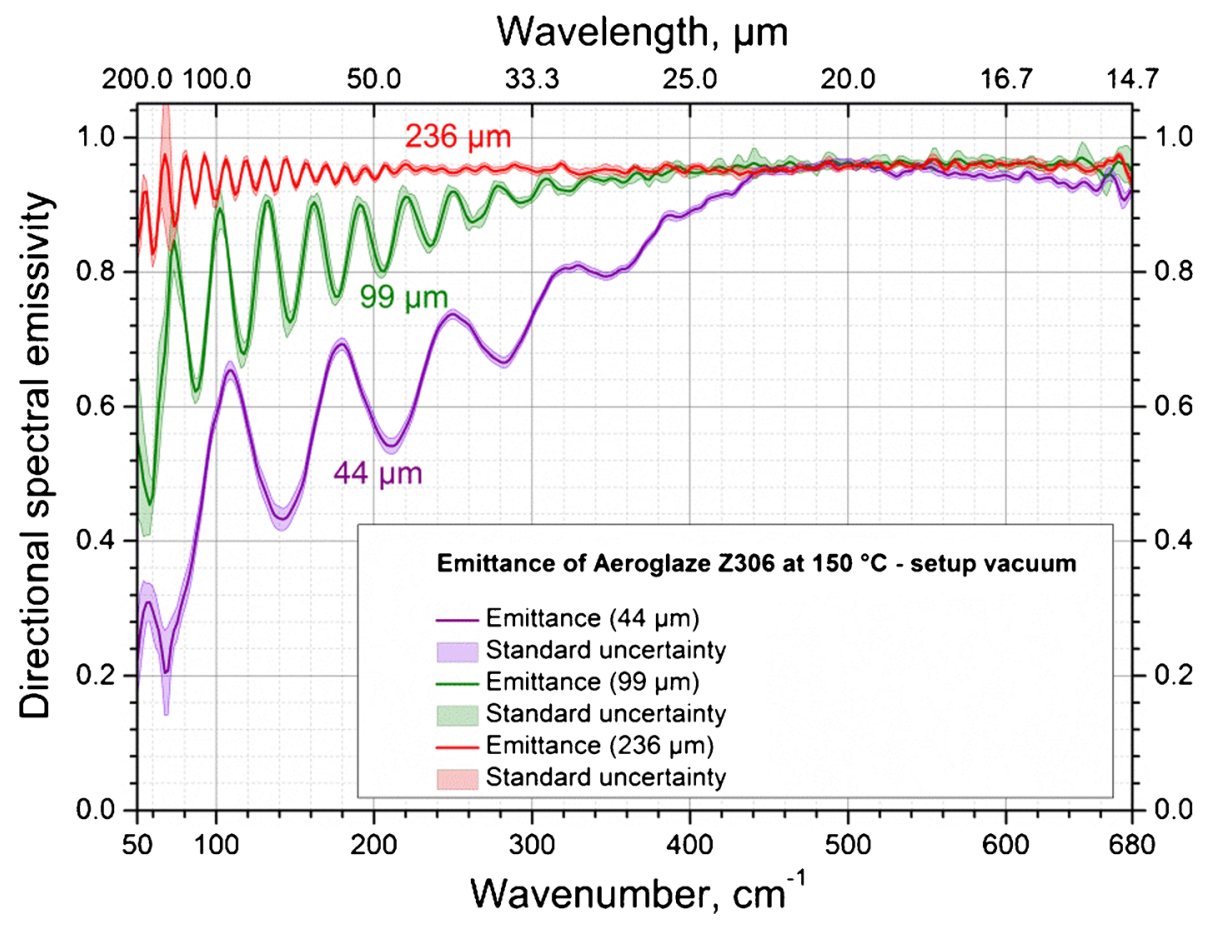

Fig. 2 Directional spectral emittance of three copper plates spray coated with Aeroglaze Z306 with thicknesses of $44 \mu \mathrm{m}, 99 \mu \mathrm{m}$ and $236 \mu \mathrm{m}$ for an angle of observation of $10^{\circ}$ to the surface normal. The standard measurement uncertainties are shown as shaded areas

three samples for an angle of observation of $10^{\circ}$ is shown in Fig 2 in combination with the standard uncertainty of the measurement, shown as shaded areas. The sample with the typical thickness used for Aeroglaze Z306 coatings of $44 \mu \mathrm{m}$ shows a significant decrease in emittance at wavelengths longer than $22 \mu \mathrm{m}$. Its emittance drops to nearly 0.2 at $200 \mu \mathrm{m}$. Furthermore, a modulation of the emittance values becomes visible. The decrease in emittance above $22 \mu \mathrm{m}$ can be explained by an increasing transparency of the coating towards longer wavelengths. This is supported by the observed modulation which typically results from multiple reflections and its interference for a partially transparent layer on a highly reflective substrate. In this case the modulation period is reciprocal of the optical thickness which is the product of thickness and refractive index. This explains also the shorter period of modulation observed for samples with a thicker coating of $99 \mu \mathrm{m}$ and $236 \mu \mathrm{m}$.

A fourth sample was prepared by brush coating Aeroglaze Z306 which resembles better the situation in the cavity of the VLTBB. Since spray coating of a monolithic cavity is not possible the VLTBB cavity was brush coated. With the brush coating a mean thickness of $88 \mu \mathrm{m}$ was obtained on the copper substrate of the sample. The measured directional spectral emittance of this sample is shown in Fig 3. The MIR part of this spectrum was determined at the setup for emissivity measurement in air [7] which is the reason for some residual water absorption artefacts. The decrease in emittance towards longer wavelengths is similar to the spray coated sample with a thickness of $99 \mu \mathrm{m}$. However, the modulation depth due to the multiple beam interference is much weaker. This results from the higher local variation in thickness within the 


\section{Wavelength, $\mu \mathrm{m}$}

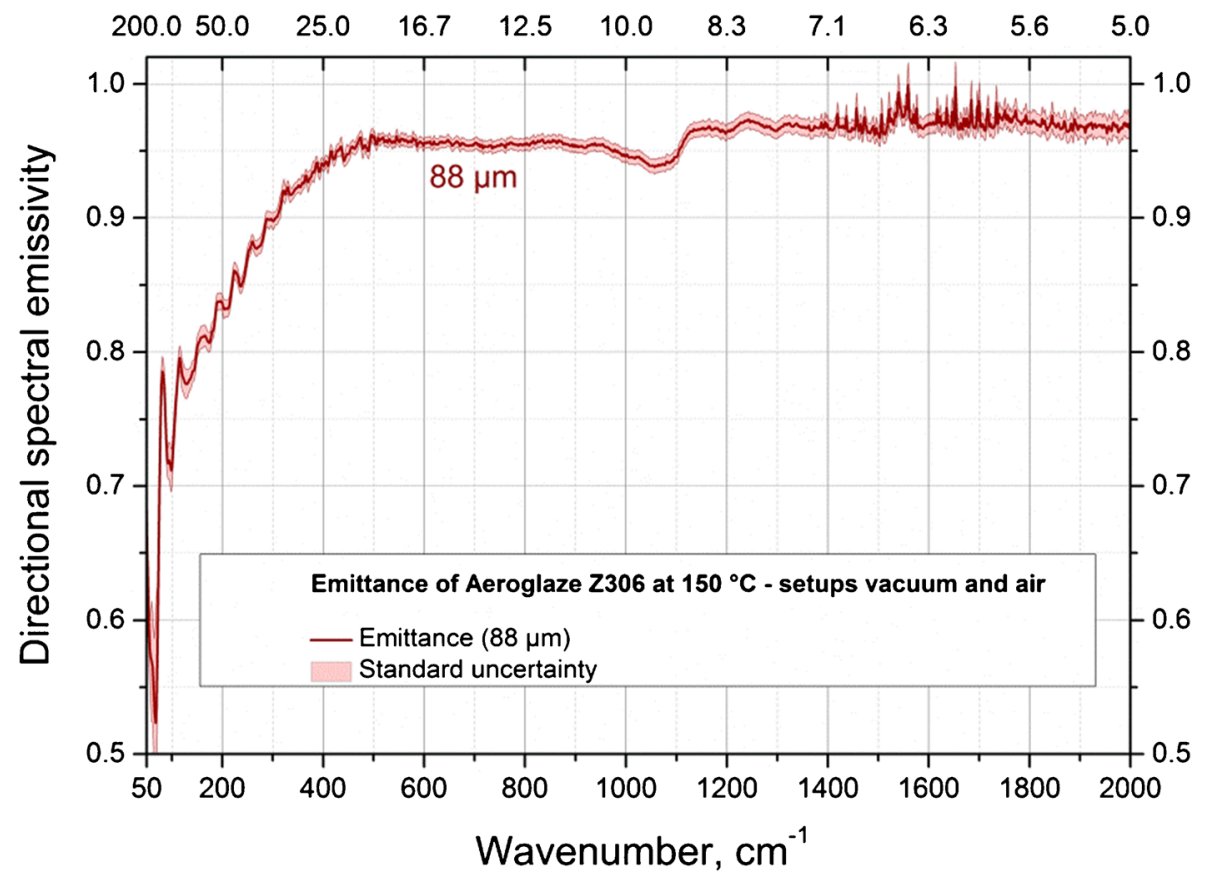

Fig. 3 Directional spectral emittance of a copper plate brush coated with Aeroglaze Z306 of a mean thickness of $88 \mu \mathrm{m}$ for an angle of observation of $10^{\circ}$ to the surface normal. The standard measurement uncertainty is shown as a shaded area

observed area of the brush coated sample with respect to the spray coated sample, while the mean thickness of both samples is comparable. This explanation can be verified by modifying the size of the observed sample area which was done for the spectral reflectance measurements of this sample and is illustrated Fig 5.

\subsection{Directional Spectral Reflectivity}

In addition to the emissivity measurements the reflectance of the Aeroglaze Z306 samples was determined with the instrumentation described in section 2.2. The directional spectral reflectance of the three copper substrates spray coated with Aeroglaze Z306 with thicknesses of $44 \mu \mathrm{m}, 99 \mu \mathrm{m}$ and $236 \mu \mathrm{m}$ is shown in Fig 4. Clearly visible is the increase in reflectance towards longer wavelength beginning at about $20 \mu \mathrm{m}$ which is in good agreement with the beginning decrease of the emittance of these samples shown in Fig 2. Also clearly visible is the modulation of the reflectance resulting from the multiple beam interference within the partly transparent layer, and its variation due to the different layer thicknesses. The reflectance of the brush coated sample which exhibits a larger local variation in its coating thickness is shown in Fig 5. Again, the increase of its reflectance corresponds well to the decrease of its emittance shown in Fig 3. To verify the assumption that a larger local variation of the sample thickness results in a weaker modulation of its reflectance, the sample was measured with two different diameters of the observed sample area. The deeper reflectivity modulation for the smaller observed 


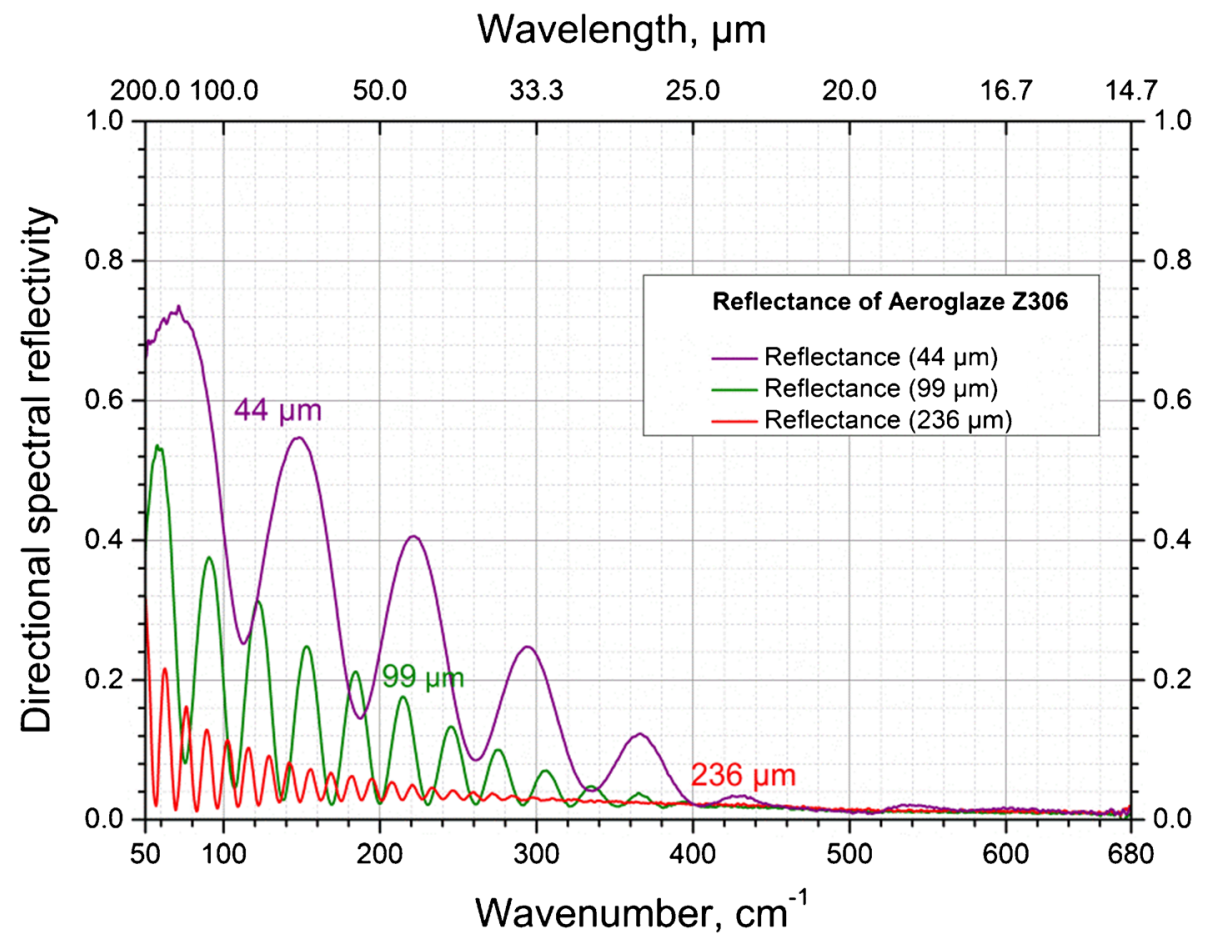

Fig. 4 Directional spectral reflectance in a $12^{\circ} / 12^{\circ}$-geometry of three copper plates spray coated with Aeroglaze Z306 with thicknesses of $44 \mu \mathrm{m}, 99 \mu \mathrm{m}$ and $236 \mu \mathrm{m}$

area, shown in Fig 6, supports the assumption that the brush coated sample has a larger local thickness variation.

\subsection{Spectral Diffusity}

The brush coated sample resembles the wall coating of the cavity of the VLTBB. Its directional spectral emittance and reflectance shown in Fig 3 and Fig 5 were used for the Monte-Carlo simulation of the effective cavity emissivity. In a first step, the spectral diffusity $D$ was calculated. It is defined as the ratio of the diffuse component of the hemispherical reflectance, $\rho_{d}$, and the sum of the diffuse component of the hemispherical reflectance and the specular component of reflectance, $\rho_{s}$.

$$
D=\frac{\rho_{d}}{\rho_{d}+\rho_{s}}
$$

The diffuse component can be calculated according to the relation $\rho_{d}=1-\varepsilon-\rho_{s}$ resulting in the following expression for the diffusity:

$$
D=\frac{1-\varepsilon-\rho_{s}}{1-\varepsilon}
$$

With the above equation the diffusity of Aeroglaze Z306 was calculated using the measured directional spectral emissivity under an angle of $10^{\circ}$ and the measured spectral reflectivity under an angle of $12^{\circ}$. They are given in Table 1 . 


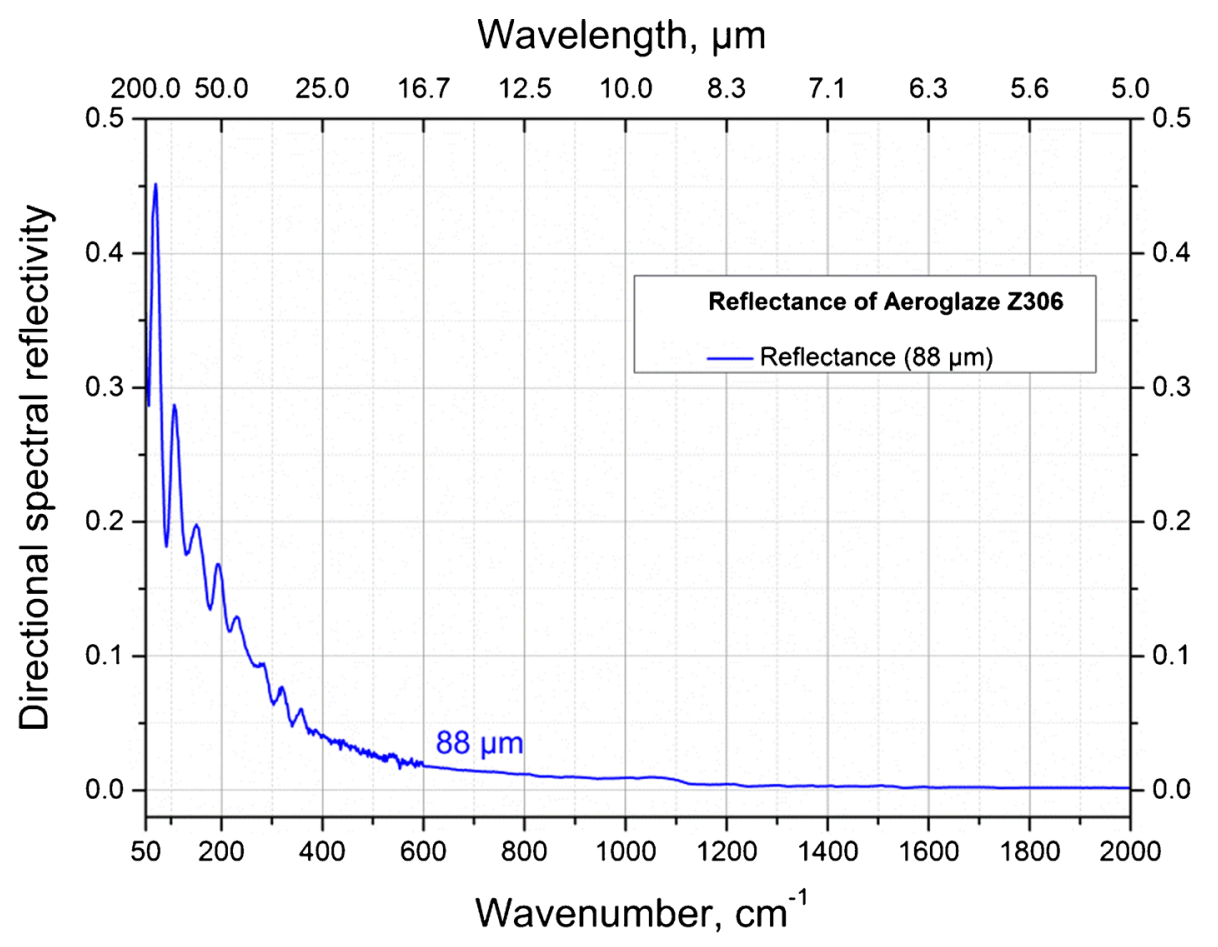

Fig. 5 Directional spectral reflectance in a $12^{\circ} / 12^{\circ}$-geometry of a copper plate brush coated with Aeroglaze Z306 with a mean thickness of $88 \mu \mathrm{m}$

\section{Monte-Carlo Simulation}

\subsection{Vacuum Variable-Low-Temperature Blackbody}

The VLTBB is a reference blackbody which provides temperature radiation from $-170{ }^{\circ} \mathrm{C}$ to $170{ }^{\circ} \mathrm{C}$ and is described in detail in [5]. A schematic view of the VLTBB is given in Fig 7. It features a liquid nitrogen cooled and electrical heated outer thermostat for a rough temperature regulation. The fine regulation is done by a three zone heating of the cavity. Each zone features a dedicated PID temperature controller. The cavity is designed for maximized temperature uniformity along its walls. Seven Platinum Resistance Temperature (PRT) sensors are used for regulation. One is located at the thermostat and six are located along the cavity, two in each heating zone with one of the two as a sensor for the respective temperature controller and the other one for independently monitoring the temperature which is used to correct the set point value of the temperature controller. Another five PRT sensors located along the cavity at different positions allow an independent monitoring of the blackbody temperature distribution.

The temperature stability of the blackbody was determined by monitoring three of its additional PRT sensors over different time intervals. Within the extended calibration uncertainty of the sensors of $30 \mathrm{mK}$ and their long term stability of $50 \mathrm{mK} /$ year one can state that the blackbody is stable and isothermal.

The cavity is a cylinder with a conical bottom of $250 \mathrm{~mm}$ in length and $40 \mathrm{~mm}$ in diameter. The angle of the bottom cone $75^{\circ}$ and the diameter of the cavity aperture is $20 \mathrm{~mm}$. The cavity is made of copper brush coated with Aeroglaze Z306. 


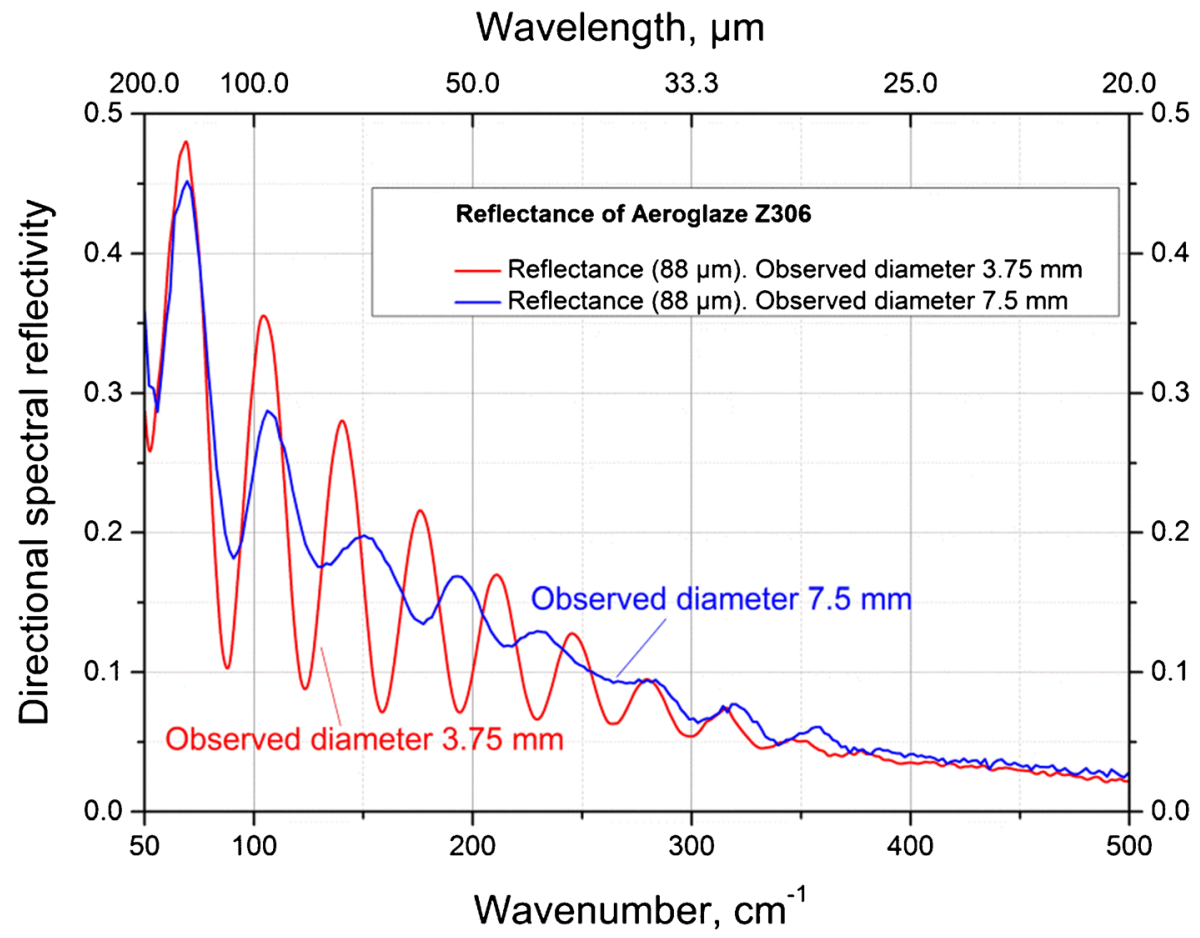

Fig. 6 Directional spectral reflectance in a $12^{\circ} / 12^{\circ}$-geometry of a copper plate brush coated with Aeroglaze Z306 with a mean thickness of $88 \mu \mathrm{m}$. Measurements within two areas of different size with diameters of $3.75 \mathrm{~mm}$ and $7.5 \mathrm{~mm}$ are shown

\subsection{Effective Spectral Cavity Emissivity}

The accurate measurement of the effective spectral cavity emissivity of a high quality blackbody is very difficult and, therefore, the computational characterization of the blackbody radiation characteristics has become a well established technique to perform this task [12]. We use the stochastic (Monte-Carlo) ray-tracing method for computer modeling of the effective cavity emissivity of the VLTBB by applying the emissivity modeling program STEEP 3 [4]. In this case the cavity geometry, the geometry of observation, the temperature homogeneity along the cavity and the spectral emissivity and diffusity of the cavity wall coating have to be known and serve as input quantities for STEEP3.

In Fig 8 the effective cavity emissivity of the VLTBB is given at five wavelengths according to the STEEP3 calculations. The calculations have been performed with the VLTBB

Table 1 Diffusity calculated from measured directional spectral reflectivity and directional spectral emissivity of a copper substrate brush coated with Aeroglaze Z306 of a mean thickness of $88 \mu \mathrm{m}$

\begin{tabular}{lllllll}
\hline Wavelength/ $\mu \mathrm{m}$ & 5 & 10 & 52 & 104 & 162 & 199 \\
\hline Directional reflectance $\left(12^{\circ}\right)$ & 0.0018 & 0.0091 & 0.1443 & 0.2621 & 0.3558 & 0.3959 \\
Directional emittance $\left(10^{\circ}\right)$ & 0.9671 & 0.9470 & 0.8305 & 0.7283 & 0.6343 & 0.5921 \\
Diffusity & 0.946 & 0.828 & 0.149 & 0.035 & 0.027 & 0.030 \\
\hline
\end{tabular}




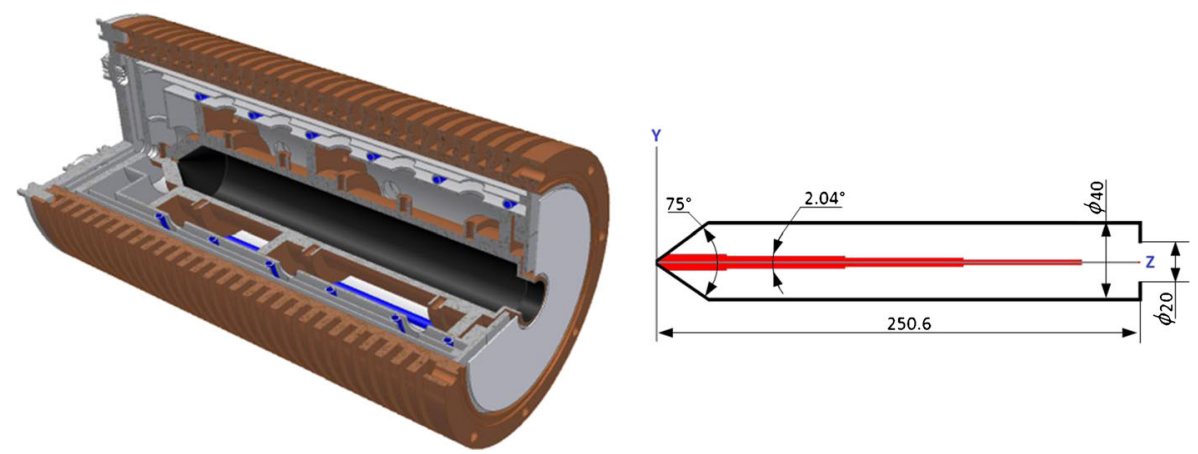

Fig. 7 On the left hand side a schematic view of the vacuum variable-low-temperature blackbody (VLTBB) of PTB's reduced background calibration facility (RBCF). On the right hand side the relevant quantities for the Monte-Carlo simulation of the effective emissivity are given: the cavity dimensions and the divergence of the observed ray bundle at the RBCF

geometry, under the typical observation conditions at the RBCF and assuming an isothermal cavity. For all calculations the measured directional spectral emittance values of Aeroglaze Z306 under an angle of observation of $10^{\circ}$ have been used (Table 1). For the data given in black squares the diffusity determined from the spectral reflectance measurements at the different wavelengths (Table 1) has been applied for an angle of $10^{\circ}$. The open circle give the calculation result if the high diffusity at $5 \mu \mathrm{m}(\mathrm{D}=0.946)$ is used under an angle of $10^{\circ}$ at all five wavelengths as input parameter to STEEP3. The diffusity has always been set to 0 for an angle of $90^{\circ}$.

While the black squares represent the best possible calculation of the effective cavity emissivity from the available input data, the open circles nicely demonstrate the strong effective of the diffusity of the wall coating on the effective cavity emissivity. The fact that the wall emissivity clearly decreases to longer wavelengths (Fig 3) can only be seen weakly for the effective emissivity in the slight decrease of the black square data points to longer wavelengths. However, when keeping the diffusity unrealistically high at longer wavelengths (open circle data) it leads to a significant decrease in effective cavity emissivity.

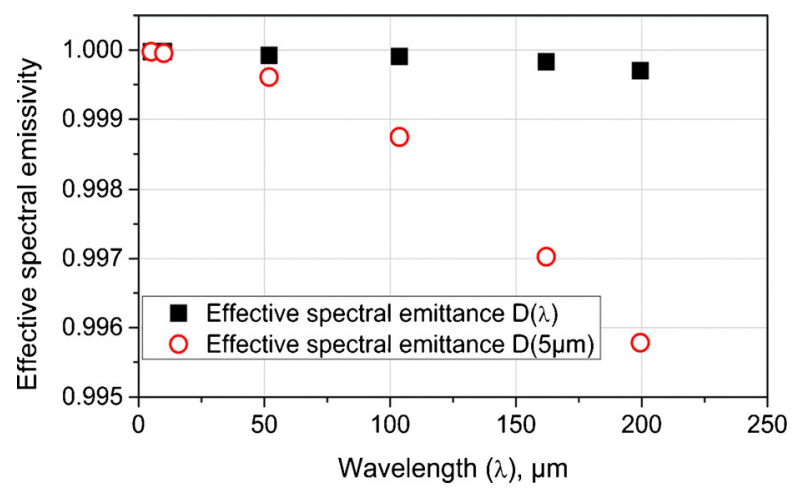

Fig. 8 Effective spectral emittance of the cavity radiator VLTBB brush coated with Aeroglaze Z306 calculated with STEEP3. For the data given in black squares the diffusity determined from the spectral reflectance measurements at the different wavelengths (Table 1) has been applied for an angle of $10^{\circ}$. The open red circles give the calculation result if the high diffusity at $5 \mu \mathrm{m}(\mathrm{D}=0.946)$ is used under an angle of $10^{\circ}$ at all five wavelengths as input parameter to STEEP3. The diffusity has always been set to 0 for an angle of $90^{\circ}$ 


\section{Summary}

Copper substrates spray coated and brush coated with Aeroglaze Z306 of different thicknesses have been characterized for their directional spectral emissivity and reflectivity. From this data the diffusity of a brush coated sample has been determined. Emissivity and diffusity values have been used to determine the effective cavity emissivity of a FIR- and THz reference blackbody via a Monte-Carlo ray tracing calculation. The calculation confirms that the blackbody has a sufficiently high emissivity up to $\mathrm{THz}$ wavelengths to be used as a standard. The influence of wall emissivity and diffusity on the effective cavity emissivity is shown.

Open Access This article is distributed under the terms of the Creative Commons Attribution License which permits any use, distribution, and reproduction in any medium, provided the original author(s) and the source are credited.

\section{References}

1. A. Steiger; B. Gutschwager, M. Kehrt, C. Monte, R. Müller, J. Hollandt, Opt. Express, OSA, 18, 2180421814, (2010) doi: 10.1364/OE.18.021804

2. T. Kleine-Ostmann, T. Schrader, M. Bieler, U. Siegner, C. Monte, B. Gutschwager, J. Hollandt, A. Steiger, L. Werner, L. Müller, G. Ulm, I. Pupeza \& M. Koch, THz Metrology, Frequenz, 62, 137-148, (2008) doi:10. 1515/FREQ.2008.62.5-6.137

3. B. Gutschwager, C. Monte, H. Delsim-Hashemi, O. Grimm J. Hollandt, Metrologia, 46, S165, (2009) doi:10. 1088/0026-1394/46/4/S06

4. V. I. Sapritsky, A. V. Prokhorov, Appl. Opt., 34, 5645-5652, (1995) doi: 10.1364/AO.34.005645.

5. S. P. Morozova, N. A. Parfentiev, B. E. Lisiansky, V. I. Sapritsky, N. L. Dovgilov, U. A. Melenevsky, B. Gutschwager, C. Monte J. Hollandt, International Journal of Thermophysics Vol. 29, 341-351, (2008) doi: $10.1007 / \mathrm{s} 10765-007-0355-\mathrm{z}$

6. C. Monte, B. Gutschwager, A. Adibekyan, M. Kehrt, F. Olschewski, J. Hollandt, 8, AIP Conf. Proc. 1552, 722 (2013) doi: 10.1063/1.4819631

7. C. Monte, J. Hollandt, High Temperatures - High Pressures, 39, 151-164, (2010)

8. A. Adibekyan, C. Monte, M. Kehrt, B. Gutschwager, J. Hollandt, Emissivity measurement under vacuum from $4 \mu \mathrm{m}$ to $100 \mu \mathrm{m}$ and from $-40{ }^{\circ} \mathrm{C}$ to $500{ }^{\circ} \mathrm{C}$ at PTB, submitted to Int. J. Thermophys., (2013)

9. C. Monte, J. Hollandt, Metrologia, 47, 172-181, (2010) doi:10.1088/0026-1394/47/2/S14

10. M. Kehrt, C. Monte, AMA Conferences 2013, Proceedings 2013, 121-123, (2013) doi:10.5162/irs2013/iP7

11. ECSS Secretariat, ESA-ESTEC Requirements \& Standards Division, European Cooperation for Space Standardization document ECSS-Q-70-25A: Space product assurance - The application of the black coating, Noordwijk, (1999)

12. A.V. Prokhorov, L.M. Hanssen, S.N. Mekhontsev, Experimental Methods in the Physical Sciences, 42, Radiometric Temperature Measurements: I. Fundamentals, 181-240, (2009) doi:10.1016/S1079-4042(09) 04205-2 DOI:10.1016/S1079-4042\%2809\%2904205-2 